\title{
Age- and sex-transcriptome analysis provide evidences for the sex biases in the pathogenesis of COVID-19 and other respiratory infectious diseases
}

Moran Gershoni ( $\sim$ gmoran@volcani.agri.gov.il )

\section{Research}

Keywords: age-related, sex-related, surfactant proteins, respiratory infectious diseases, COVID-19

Posted Date: July 17 th, 2020

DOI: https://doi.org/10.21203/rs.3.rs-43708/v1

License: (c) (i) This work is licensed under a Creative Commons Attribution 4.0 International License.

Read Full License 
3 Moran Gershoni ${ }^{1^{*}}$

$7 \quad$ Keywords

8 age-related, sex-related, surfactant proteins, respiratory infectious diseases, COVID-19 


\section{Abstract}

11 Age and sex were shown to affect the prevalence and the manifestation of many respiratory infectious

12 diseases. These can be attributed to age and sex related alterations in the immune system and in the

13 lung functions. Since the outbreak of COVID-19, epidemiological studies consistently report that age and

14 sex are major risk factors in both morbidity and mortality due to COVID-19. Thus, understanding age and

15 sex dependent gene expression in the lung and in the immune system can provide mechanical evidences

16 with respects to sex related higher risk of elderly to develop severe complications in respiratory

17 infectious diseases. In this context, sex- and age- transcriptome analysis from hundreds of lung and

18 blood samples, revealed significant downregulation of the lung surfactant and blood innate immune

19 genes, that occur predominantly in elderly men. Depletion in lung surfactant leads to enhanced injury of

20 alveolar epithelium and fibrotic destruction, and recruitment of the innate immune system is essential

21 to control infection of new pathogens like SARS-CoV-2. Interestingly, surfactant proteins, which protect

22 the lung from infection, are co-produced with the SARS-CoV-2 host receptor- ACE2, by the AT2 cells.

23 Thus, infection by SARS-CoV-2 is expected to lead to decline in AT2 cells and a loss of surfactant

24 proteins, especially in elderly men.

25 
27 Many infectious diseases, such as influenza and pneumonia, are more common in the elderly, and are associated with poor outcome. Partially, these can be attributed to age related alterations in the immune system, including modifications in several component of the innate immune system, like alterations in secretion and response to cytokines[1]. Sex differences were also described for the manifestations of many infectious diseases, including infections caused by viruses. These sex differences

32 were shown to be associated with differential immune regulation[2,3]. A recent study in mice suggested

33 that the stronger immune response of females might be the results of more activated innate immune 34 pathways prior to infection[4]. In December 2019, a new lethal infectious respiratory disease emerged in Wuhan China[5,6] . This coronavirus disease 19 (COVID-19) has, so far, caused more than 10 million confirmed cases (28 June 2020[7]). Sequence analysis found a novel coronavirus, SARS-CoV-2, which is

37 closely related to SARS-CoV, as the cause of the new lung disease[8]. Early epidemiologic analysis of more than 70 thousand cases in China indicates that about $80 \%$ of COVID-19 patients experience only mild symptoms, but some $5 \%$ developed severe pneumonia that in many cases leads to death. The overall case fatality rate (CFR) was estimated as 2.3 . In this analysis three notable trends were observed[9]:

1) The male CFR was almost twice as high than of females (2.8 vs 1.7 respectively)

2) CFR sharply increases as a function of age, where CFR for patients below 50 is less than 0.4 and approximately 15 for patients above 80

47 Korea, where an intensive policy of testing for outbreak monitoring was implemented[10], the local CDC reported on more than $1,250,000$ tests and more than 12,700 confirmed cases, with an overall 2.22 CFR. 
At that time about $58 \%$ of the confirmed cases were females but their CFR was reported to be 1.8 ,

50 compared to the male CFR of 2.77. The differences in the age-related CFR were even higher than in

51 China, with less than 0.2 for patients younger than 50 , and 25 for patients older than 80 (28 June

52 2020[11]). Similar age and sex biases were also reported by the New York City health department[12].

53 UK data from 4000 COVID-19 patients that were admitted to intensive care units since the outbreak

54 showed an almost 3-fold more male than female cases in critical condition $(72.5 \%$ and $27.5 \%$

55 respectively). This work also reported an age-related survival rate for patient in critical condition ranging

56 from $75 \%$ for young adults to only $27 \%$ for patients older than 75 . Such differences have not been

57 observed in pneumonia caused by other viral infections[13]. A recent study that summarized data from

5838 countries reported average male CFR to be 1.7-fold higher than average female CFR[14]. Finally, a

59 large study from France, that included serological and clinical data, reported age and male bias for

60 hospitalization, intensive care units (ICU) admittance, and mortality rate. This study also reported that

61 although this sex bias occurs at most age intervals, the male to female mortality rate ratio increases with

62 age, and was almost 3 fold for patients older than $80[15]$. Molecular and clinical studies have reported

63 that, of all confirmed COVID-19 cases, up to $20 \%$ will develop pneumonia, and of them some will

64 develop acute respiratory distress syndrome (ARDS), primarily patients older than 65[16].

65 The complete genome sequence of SARS-COV-2 was made public in early January 2020

66 (http://virological.org/). Initial sequence comparisons revealed that SARS-CoV-2 is approximately $79 \%$

67 identical to SARS-CoV at the nucleotide level[17]. Structural studies and biochemical experiments show

68 SARS-CoV-2 virions to be optimized for binding to the human ACE2 receptor ([17]and references within).

69 An additional study found that, like SARS-CoV, SARS-CoV-2 spread also depends on the proteolytic

70 activity of human TMPRSS2 protease[18]. Thus, tissues expressing both genes are prone to infection and

71 virus replication, as demonstrated in the respiratory and digestive systems[19]. 
72 Taken together, the above evidences suggesting that age-related sexual differences that exist prior to

73 infection in the lung and in the immune system might explain age- and sex- biased pathogenesis

74 observed in several infectious disease and specifically in COVID-19. Therefore, the hypothesis of this

75 study is that age and sex related biological factors, specifically in the lung and the immune system, are

76 the cause for the high susceptibility of elderly and male patients who contract SARS-Cov-2, to develop

77 ARDS and a pathological inflammatory condition that leads to a higher mortality rate. Identification of

78 genes that have differential expression (DE), specifically between elderly males and elderly females, may

79 provide evidences for the age and sex biased pathogenesis of COVID-19 and other respiratory infectious

80 diseases. To test this hypothesis, an age and sex differential gene expression analysis from hundreds of

81 publicly available lung and blood samples, followed by comprehensive bioinformatics functional

82 annotation and disease association, was carried out. These analyses revealed a significant age-related

83 decline in the expression of the lung surfactant proteins (SFTPs) in men. In addition, downregulation of

84 dozens of genes associated with the innate immune system in the blood, including the Toll-Like

85 Receptor 4 (TLR4) signaling pathway, was found. All these differences were found to be predominant in

86 older men as compared to older women. Deficiency in surfactant proteins genes leads to idiopathic

87 pulmonary fibrosis and incomplete repair of injured alveolar epithelium, mainly in older adults[20,21].

88 The recruitment of the innate immune system may be a determinant for efficient protection of the host,

89 in the case of SARS-CoV-2 infection, since this virus is newly introduced to the immune system and there

90 is no pre-existing adaptive immune memory. Finally, in attempting to provide genetic markers that can

91 pinpoint risk factors associated with the identified age- and sex- related genes, a screening of public

92 databases identified eQTLs and GWAS QTLs that associated with the expression and malfunction of

93 these genes. Such genetic markers should be further evaluated to assess their clinical significance in

94 COVID-19, specifically in elderly.

\section{Material and methods}


Data acquisition. RNA-seq data, samples and donor annotations, genes annotations, and eQTLS

97 information were obtained from the GTEx project version 8[22]. The population variation data and the minor alleles frequencies (MAFs) were obtained from the gnomAD database[23] ( $N=160,000)$.

Analysis of sex and age differential transcriptome. Age and sex differential expression was calculated as previously described[24]. First, all the samples were annotated according to the tissue and the donor's age and sex. Then, all the samples from the Lungs ( $\mathrm{N}_{\text {all }}=578 ; \mathrm{N}_{\text {men }}=395 \mathrm{~N}_{\text {women }}=183$ ) and Blood ( $\mathrm{N}_{\text {all }}=755$; $\mathrm{N}_{\text {men }}=501 \mathrm{~N}_{\text {women }}=183$ ) were further obtained from the full dataset, and divided into four groups according to age (older or younger than 60 ) and sex. Only protein coding genes were included in the analysis. The NOISeqBIO $[25,26]$ algorithm was then used to compare Transcripts Per Million (TPM) expression values between the following comparisons:

\begin{tabular}{|l|l|}
\hline Men younger than 60 & Men older than 60 \\
\hline Women younger than 60 & Women older than 60 \\
\hline Women older than 60 & Men older than 60 \\
\hline
\end{tabular}

A NOIseqBIO probability cutoff of 0.95 was used to identify genes with significant differential

107 expression, as this cutoff value was shown to correct for multiple testing in similar datasets[27].

108 Differentially expressed genes were annotated and enrichment analysis was performed using the

109 GeneAnalytics server, which can identify gene enrichment for several terms and data sources, including 110 diseases, biological pathways, GO terms, and tissue expression[28]. To test Co-localization of suspected

111 eQTLs, genomic variants which were reported to be associated with diseases and traits relevant to

112 COVID-19 (e.g., pulmonary diseases) were obtained from the GWAS atlas[29].

\section{Results}

114 The working hypothesis of this study is that significant age-related differences in genetic expression 115 between men and women in the respiratory and the immune systems can reveal contributors to the age 
and sex biases pathogenesis of COVID-19 and other respiratory infections. The immune system can be

117 sufficiently monitored by profiling blood transcriptome[30] and the lung represent the most affected 118 tissue in ARDS[16]. Thus, to test the working hypothesis, the following analyses were performed-

119 Differential expression in blood and lung between: Women older than 60 versus women younger than

12060 ; men older than 60 versus men younger than 60; men older than 60 versus women older than 60.

121 The results of these analyses are shown in supplementary tables 1-6, and the number of differentially

122 expressed (DE) genes identified in these analyses within and between the tested conditions are

123 summarized in figure 1 . Overall, about 3000 and 2000 age related DE (ADE) genes were identified in the

124 blood and lungs, respectively. The vast majority of these genes showed age-related differential

125 expression in men. $212 / \sim 3000$ of the blood genes and $20 / \sim 2000$ of the lung genes that have significant

126 ADE also showed significant DE between older men to older women (figures 1a and 1b). Such genes are

127 the main candidates to contribute to the age- and sex- related manifestation that have been observed in

128 COVID-19. In order to assess the possible role of these ADE genes in ARDS and COVID-19, a functional

129 analysis was carried out and the enrichment of these ADE genes to biological pathways, diseases,

130 phenotypes and GO terms was computed using the GeneAnalytics tool[28]. The results of these analyses

131 are presented in supplementary tables 7-12 and summarized in tables 1 and 2 . Genes that undergo

132 significant downregulation in the blood of old men compared to old women are mainly associated with

133 the innate immune system response for viral infection (supplementary tables 7-9, table 1 and figure 2).

134 Among these genes are IFITM1 and IFITM2 which are part of the IFN Alpha/beta Signaling pathway

135 (table 1 and figure 2) and were previously shown to be important for the resistance against RNA viruses,

136 including SARS-CoV[31]. Another innate immune response pathway that was found to be downregulated

137 is the Tol-Like-Receptor 4 (TLR4) signaling pathway (table 1, figure 2). Results from a previous study

138 showed that SARS-CoV membrane protein may function as a cytosolic pathogen-associated molecular

139 pattern (PAMP) which stimulates IFN- $\beta$ production by activating TLR-related TRAF3-independent 
signaling cascade[32]. In addition, several genes like SERPINB1 that are part of the innate immune

141 response are also associated with lung diseases (table 1, figure 2). Interestingly, SERPINB1 was reported

142 to have a protective immunomodulatory activity that prevents lung epithelial tissue injury[33]. Genes

143 that undergo significant age-related downregulation in male lungs are specifically associated with

144 pulmonary surfactant metabolism dysfunction and pulmonary fibrosis (supplementary tables 10-12,

145 table 2, figure 3). The surfactant genes SFTPB and SFTPC have significant additional downregulation in

146 old men compared to old women (supplementary tables 4-6, table 2 and figure 3). Functional deficiency

147 of these genes leads to fatal neonatal respiratory distress and pulmonary alveolar proteinosis[34]. Age

148 related downregulation of lung surfactant could be the results of reduced expression, but could also be

149 due to alteration in the tissue cell's composition. SFTPs genes were shown to be co-expressed with

150 ACE2, the SARS-CoV-2 host receptor, by the alveolar type II (AT2) cells. Thus, changes in AT2 cells

151 distribution in the lung tissue is expected to be reflected by ACE2 age and sex related expression like the

152 SFTPs genes. However, ACE2 expression analysis revealed no such age or sex related differences in the

153 lungs (supplementary tables 4-6 and figure 3). To this point, the DE analyses have pointed to the

154 association of two systems with the high age and sex related susceptibility to develop severe

155 complications in respiratory infectious diseases including in COVID-19: the innate immune response and

156 the lung surfactant metabolism. Thus, one can ask if the likelihood of having downregulation in these

157 pathways is independent or tends to be correlated. To answer this, blood and lung samples from the

158 same donors were obtained $(n=154)$, and the Pearson correlation co-efficiency values were calculated

159 between the blood innate immune system ADE genes $(n=82)$ and the lung SFTPs genes expression. This

160 analysis revealed that the expressions of the lung SFTPs genes are highly correlated with each other $(r>$

161 0.9) and, more importantly, the expression of the SFTPs genes tend to be correlated with most blood

162 innate immune genes. Specifically, a significant high correlation between SFTPB, TLR5 and SERPINB1 $(r=$

$1630.76 ; p<0.00001$, figure 4) was found. These results suggest that patients with low expression of the 
164 blood innate immune ADE genes (that can be detected from blood samples) also tend to have reduced

165 SFTPs expression. Finally, screening for genetic variants that associated with SFTPs gene dysfunction and 166 expression can be useful for further evaluation of the clinical significance of these lung genes in COVID-

16719 and other respiratory diseases. To this end, the GTEx eQTL database was screened to identify lung 168 eQTLs that significantly alter the expression of the SFTPs genes. In addition, the GWAS atlas[29] was 169 screened to identify variants associated with relevant diseases and phenotypes (e.g. pulmonary diseases 170 and pulmonary functions) and are co-localized to the same eQTLs loci (+/- 0.5Mb). Such co-localized 171 variants are prime candidates to undergo further evaluation and assessment of clinical significance. The 172 results of these screens are summarized in table 3.

\section{Discussion}

174 Age related physiological changes are involved in impairing respiratory infections and its poor outcomes 175 in the elderly. This high vulnerability can be attributed to modified immune response and to other 176 alterations also occur in the lung as a consequence of the ageing process, which gradually lead to a 177 decline in lung function[35]. Sex related biological differences were also shown to affect the prevalence 178 and the course of many diseases. Many of these differences can be relate to differential gene 179 expression[36]. previous study found that in many cases, this sex differential expression is age 180 dependent[24]. Previously, a meta-analysis that preformed on more than 80 studies reported that males 181 are more commonly affected with infections in the lower respiratory tract, and that the course of most

182 respiratory diseases is more severe in males than in females, leading to higher mortality in males, 183 especially in community-acquired pneumonia[37].

184 One of the open questions since the outbreak of COVID-19 regards the causes for the age and sex biases 185 in the morbidity and mortality rates due to SARS-CoV-2 infection. Clinical, epidemiological, and 186 pathological evidences from COVID-19 studies demonstrate the involvement of the immune and the 
respiratory systems, in an age and sex dependent manner, in the pathophysiology of the disease[15,38].

188 The increased sex- and age- related susceptibility is likely a result of naturally occurring differences between the sexes that express upon the properties of the SARS-CoV-2 infection. Sex related gene expression in the immune system was previously shown to affect the response to a pathogen prior to

191 infection[4]. In this context, the age and sex differential transcriptome of lungs and blood was analyzed 192 from hundreds of samples. These analyses revealed hundreds of ADE genes, mainly in men. The differences in the amount of ADE genes in these tissues between men and women could be explained by 194 the differences in the sample sizes, which are much larger for men (for lung $N_{\text {men }}=395 ; N_{\text {women }}=183$, and 195 for blood $N_{m e n}=501 ; N_{\text {women }}=183$ ). However, comparing the fold changes in gene expression between old to young, out of 19,179 protein coding genes that were analyzed, 745 and 225 genes have an average of

197 1.5-fold change or greater, in the lung of men versus women, respectively. This suggests that while the 198 differences in the number of identified ADE genes between men and women was likely affected by the 199 sample sizes, it should also be attributed to the biological differences between men and women in the 200 aging process. Functional analysis of the ADE genes within and between men's and women's blood 201 tissue revealed that the most affected age-related pathway in men is the innate immune system (table 202 1). Most of the blood ADE genes that associated with the innate immune system have a significantly 203 reduced expression, which suggests a male age-related down regulation of the innate immune system.

204 Functional analysis of ADE genes between elderly men and elderly women found the enrichment of a set 205 of 82 genes of the innate immune system, which all had significantly reduced expression in elderly men 206 as compared to elderly women. These findings suggest that women's innate immune system is less 207 affected by age. Some of the DE genes between elderly men to elderly women are specifically associated 208 with the TLR4 signaling pathway (e.g., TLR2, TLR4 and TLR5, table 1 and figure 2). The innate immune 209 response is the first mechanism of defense against infections. TLRs are important for the development 210 and activation of innate immunity. The role of TLR4 is typically part of the response to the LPS of gram- 
211 negative bacteria[39]. However, a recent study suggested that cell surface TLR4 is most likely to be

212 involved in recognizing SARS-CoV-2 and to inducing inflammatory responses, and thus plays a crucial

213 role in the initial virus-induced inflammatory consequences associated with COVID-19[40]. Another

214 group of blood downregulated DE genes between elderly women to elderly men, are directly associated

215 with lung diseases. Among these genes are SERPBINA1 and SERPBINB1. These genes have a serine

216 protease elastase activity, and deficiency of serpinB1 has been shown to decrease the survival rate and

217 increases morbidity associated with murine pulmonary influenza. This was shown to be likely due to

218 enhanced injury of lung epithelial and the failing to downregulate pro-inflammatory cytokine

219 production, similar to the pathological finding in COVID-19 and other respiratory infectious diseases[41].

220 Mouse models found that the regulation of pulmonary innate immunity by SERPINB1 is an essential

221 process in the host response to infection, accomplished by controlling the recruitment of neutrophils to

222 the infected lung. SERPINB1 deficiency leads to overproduction of pro-inflammatory cytokines that are

223 associated with fatal outcome of human influenza[41,42]. Mutations in the SERPINA1 gene are known to

224 cause Alpha-1 antitrypsin (AAT) deficiency. With insufficient functional AAT, neutrophil elastase destroys

225 alveoli and typically causes age-related lung disease[43-45].

226 Functional analysis of the lung ADE genes within and between men and women reveals that the most

227 affected age-related pathways differing between men and women are the surfactant metabolism and

228 pulmonary fibrosis (table 2), including all the SFTPs genes. The surfactant proteins, encoded by the

229 SFTPs genes, are expressed and produced by the AT2 cells and are essential to maintaining lung

230 homeostasis. Deficiency in these genes has been found to cause idiopathic pulmonary fibrosis and

231 incomplete repair of injured alveolar epithelium, mainly in older adults[20,21]. Interestingly, SARS-CoV-2

232 host receptor, ACE2, was also found to be expressed by AT2 cells[46]. Investigation of histopathology

233 sections from COVID-19 patients' discovered SARS-CoV-2 particles in AT2 epithelia, which was confirmed

234 by RT-PCR[47]. Thus, the lung AT2 cells which are likely the SARS-CoV-2's target are also the producers 
of the surfactant proteins. Depletion of AT2 cells due to SARS-CoV-2 infection, or other pathogens that

236 targeting the AT2 CELLS, is therefore expected to cause surfactant deficit, which has previously been

237 shown to be associated with incomplete repair of injured alveolar epithelium and fibrotic

238 elimination[20]. Similar to surfactant deficit, the main histological finding from autopsies taken from

239 lungs of COVID-19 patients includes injury to the alveolar epithelial cells and fibrosis[48]. Altogether,

240 these results show that the basal expression levels of the SFTPs genes in men older than 60 tend to be

241 very low (figure 3, Supp. Table 4-6) as compared to younger men and women in all ages. Thus, older

242 men are expected to be much more vulnerable to respiratory infection, specifically by pathogen that

243 infect the lung AT2 cells, like in SARS-CoV-2 infection, due to the additional depletion of AT2 cell and loss

244 of surfactant proteins.

\section{Conclusions}

246 Altogether, the findings of this study provide evidence for the high prevalence and poor outcome of

247 elderly, specifically of males, to respiratory infectious diseases, and can illuminate a possible sex biased

248 mechanism in COVID-19 pathogenesis: In the absence of adaptive immune memory, downregulation of

249 the innate immune system in the elderly, especially in old men, suppresses their initial response to

250 SARS-CoV-2 infection, likely through genes such as the TLRs. This, in turn, reduces virus clearance and

251 increases the susceptibility of old men to developing pulmonary infection. The SARS-CoV-2 target host

252 cells in the lungs are the AT2 cells, the producers of the surfactant proteins. The initial expression levels

253 of the surfactant genes tend to be much lower in old men. Thus, additional decline of AT2 cells due to

254 viral infection will abolish surfactant proteins and thus promote massive injury of the alveolar

255 epithelium and fibrosis. This pathological process enhances hyper-inflammation that should be

256 modulated by additional components of the innate immune system, like the SERPINs genes. However,

257 the expression of these genes is the lowest in elderly men. Since the expression of the innate immune 
258 ADE genes and the SFTPs genes tend to be correlated, it is likely that subgroup of elderly male COVID-19

259 patients, will have an overall poor response to SARS-CoV-2 infection in both systems.

260 Ethics approval and consent to participate

261 Not applicable

262 Consent for publication

263 Not applicable

264 Availability of data and materials

265 All the data is publicly available. The GTEx Analysis V8 RNA-seq and eQTL data are available on the GTEx

266 portal (http://www.gtexportal.org/home/datasets).

\section{Competing interests}

268 The author declares no competing interest

$269 \quad$ Funding

270 This work was partially supported by a grant from the Israeli Science Foundation (ISF 946/19 to M.G.).

271 The grant foundations had no competing interest.

\section{Authors' contributions}

273 M.G. designed the study, performed the analyses, performed data mining and wrote the paper

\section{Acknowledgements}

275 I want to thank Shmuel Pietrokovski for helpful discussion and for pointing me to relevant literature. I

276 want to thank Aviva Isaacs for critical reading of the manuscript.

\section{References}


1. Gavazzi G, Krause KH. Ageing and infection. Lancet Infect. Dis. Lancet Publishing Group; 2002. p. 65966.

2. Klein SL, Jedlicka A, Pekosz A. The Xs and $Y$ of immune responses to viral vaccines [Internet]. Lancet

281 Infect. Dis. 2010 [cited 2020 Jul 7]. p. 338-49. Available from:

282 https://www.sciencedirect.com/science/article/pii/S1473309910700499

3. Fish EN. The X-files in immunity: Sex-based differences predispose immune responses [Internet]. Nat. 284 Rev. Immunol. 2008 [cited 2020 Jul 7]. p. 737-44. Available from: https://www.nature.com/articles/nri2394

4. Gal-Oz ST, Maier B, Yoshida H, Seddu K, Elbaz N, Czysz C, et al. ImmGen report: sexual dimorphism in 287 the immune system transcriptome. Nat Commun [Internet]. Nature Publishing Group; 2019 [cited 2020 Jul 7];10:1-14. Available from: https://doi.org/10.1038/s41467-019-12348-6

5. Huang C, Wang Y, Li X, Ren L, Zhao J, Hu Y, et al. Clinical features of patients infected with 2019 novel coronavirus in Wuhan, China. Lancet [Internet]. 2020 [cited 2020 Apr 19];395:497-506. Available from:

291 https://www.sciencedirect.com/science/article/pii/S0140673620301835

292 6. Wang C, Horby PW, Hayden FG, Gao GF. A novel coronavirus outbreak of global health concern

293 [Internet]. Lancet. 2020 [cited 2020 Apr 19]. p. 470-3. Available from:

294 https://www.thelancet.com/journals/lancet/article/PIIS0140-6736(20)30185-9/fulltext

295 7. COVID-19 situation reports [Internet]. [cited 2020 Apr 19]. Available from:

296 https://www.who.int/emergencies/diseases/novel-coronavirus-2019/situation-reports

297 8. Zhu N, Zhang D, Wang W, Li X, Yang B, Song J, et al. A novel coronavirus from patients with 298 pneumonia in China, 2019. N Engl J Med. Massachussetts Medical Society; 2020;382:727-33.

299 9. China CDC. METHOD Study Design •. cdn.onb.it [Internet]. 2020 [cited 2020 Apr 19];5. Available from: 
301 10. Al-Rousan N, Al-Najjar H. Data Analysis of Coronavirus CoVID-19 Epidemic in South Korea Based on

302 Recovered and Death Cases. J Med Virol [Internet]. 2020 [cited 2020 Apr 19]; Available from:

303 http://www.ncbi.nlm.nih.gov/pubmed/32270521

304 11. List | Press Release | News Room : KCDC [Internet]. [cited 2020 Apr 19]. Available from:

305 https://www.cdc.go.kr/board/board.es?mid=a30402000000\&bid=0030

306 12. COVID-19: Data - NYC Health [Internet]. [cited 2020 Apr 19]. Available from:

307 https://www1.nyc.gov/site/doh/covid/covid-19-data.page\#download

308 13. ICNARC - Reports [Internet]. [cited 2020 Apr 19]. Available from: https://www.icnarc.org/Our309 Audit/Audits/Cmp/Reports

310 14. Scully EP, Haverfield J, Ursin RL, Tannenbaum C, Klein SL. Considering how biological sex impacts

311 immune responses and COVID-19 outcomes. Nat. Rev. Immunol. Nature Research; 2020.

312 15. Salje H, Tran Kiem C, Lefrancq N, Courtejoie N, Bosetti P, Paireau J, et al. Estimating the burden of

313 SARS-CoV-2 in France. Science (80- ). American Association for the Advancement of Science (AAAS);

$314 \quad 2020 ;$ eabc3517.

315 16. Mason RJ. Pathogenesis of COVID-19 from a cell biology perspective [Internet]. Eur. Respir. J.

316 European Respiratory Society; 2020 [cited 2020 Jun 23]. p. 2000607. Available from:

317 http://erj.ersjournals.com/lookup/doi/10.1183/13993003.00607-2020

318 17. Andersen KG, Rambaut A, Lipkin WI, Holmes EC, Garry RF. The proximal origin of SARS-CoV-2. Nat 319 Med. Springer Science and Business Media LLC; 2020;26:450-2. 
321 Entry Depends on ACE2 and TMPRSS2 and Is Blocked by a Clinically Proven Protease Inhibitor. Cell. Cell

322 Press; 2020;181:271-280.e8.

323 19. Wölfel R, Corman VM, Guggemos W, Seilmaier M, Zange S, Müller MA, et al. Virological assessment

324 of hospitalized patients with COVID-2019. Nature. Springer Science and Business Media LLC; 2020;1-10.

325 20. Li Y, Wu Q, Sun X, Shen J, Chen H. Organoids as a Powerful Model for Respiratory Diseases [Internet].

326 Stem Cells Int. 2020 [cited 2020 Jun 23]. Available from:

327 https://www.hindawi.com/journals/sci/2020/5847876/

328 21. Wang Y, Kuan PJ, Xing C, Cronkhite JT, Torres F, Rosenblatt RL, et al. Genetic Defects in Surfactant

329 Protein A2 Are Associated with Pulmonary Fibrosis and Lung Cancer. Am J Hum Genet. 2009;84:52-9.

330 22. Melé M, Ferreira PG, Reverter F, DeLuca DS, Monlong J, Sammeth M, et al. The human

331 transcriptome across tissues and individuals. Science (80- ). American Association for the Advancement

332 of Science; 2015;348:660-5.

333 23. Karczewski KJ, Francioli LC, Tiao G, Cummings BB, Alföldi J, Wang Q, et al. Variation across 141,456

334 human exomes and genomes reveals the spectrum of loss-of-function intolerance across human

335 protein-coding genes. bioRxiv [Internet]. Cold Spring Harbor Laboratory; 2019 [cited 2020 Apr

336 19];531210. Available from: https://www.biorxiv.org/content/10.1101/531210v3

337 24. Gershoni M, Pietrokovski S. The landscape of sex-differential transcriptome and its consequent

338 selection in human adults. BMC Biol. 2017;15.

339 25. Tarazona S, García-Alcalde F, Dopazo J, Ferrer A, Conesa A. Differential expression in RNA-seq: A

340 matter of depth. Genome Res. Cold Spring Harbor Laboratory Press; 2011;21:2213-23.

341 26. Tarazona S, Furió P, Ferrer A, Conesa A. NOISeq: An R package for differential expression in RNA-Seq

342 using biological replicates. 2013. 
27. Tarazona S, Furió-Tarí P, Turrà D, Di Pietro A, Nueda MJ, Ferrer A, et al. Data quality aware analysis of

344 differential expression in RNA-seq with NOISeq R/Bioc package. Nucleic Acids Res [Internet]. 2015 [cited

3452020 Apr 19];43. Available from: https://academic.oup.com/nar/article-abstract/43/21/e140/2468096

346 28. Fuchs SBA, Lieder I, Stelzer G, Mazor Y, Buzhor E, Kaplan S, et al. GeneAnalytics: An Integrative Gene

347 Set Analysis Tool for Next Generation Sequencing, RNAseq and Microarray Data. Omi A J Integr Biol.

348 Mary Ann Liebert Inc.; 2016;20:139-51.

349 29. Watanabe K, Stringer S, Frei O, Umićević Mirkov M, de Leeuw C, Polderman TJC, et al. A global

350 overview of pleiotropy and genetic architecture in complex traits. Nat Genet. Nature Publishing Group;

$351 \quad 2019 ; 51: 1339-48$.

352 30. Chaussabel D, Pascual V, Banchereau J. Assessing the human immune system through blood

353 transcriptomics [Internet]. BMC Biol. BioMed Central; 2010 [cited 2020 Jun 28]. p. 1-14. Available from:

354 https://link.springer.com/articles/10.1186/1741-7007-8-84

355 31. Huang IC, Bailey CC, Weyer JL, Radoshitzky SR, Becker MM, Chiang JJ, et al. Distinct patterns of

356 IFITM-mediated restriction of filoviruses, SARS coronavirus, and influenza A virus. PLoS Pathog. $2011 ; 7$.

357 32. Wang Y, Liu L. The membrane protein of severe acute respiratory syndrome coronavirus functions as

358 a novel cytosolic pathogen-associated molecular pattern to promote beta interferon induction via a toll-

359 like-receptor-related TRAF3-independent mechanism. MBio [Internet]. American Society for

360 Microbiology; 2016 [cited 2020 Jun 23];7. Available from: https://mbio.asm.org/content/7/1/e01872-15

361 33. Zhao P, Hou L, Farley K, Sundrud MS, Remold-O'Donnell E. SerpinB1 regulates homeostatic

362 expansion of IL-17 $+\gamma \delta$ and CD4 + Th17 cells. J Leukoc Biol [Internet]. 2014 [cited 2020 Jun 23];95:521-

363 30. Available from: https://jlb.onlinelibrary.wiley.com/doi/abs/10.1189/jlb.0613331

364 34. Wallot M, Wagenvoort C, Demello D, Müller KM, Floros J, Roll C. Congenital alveolar proteinosis 
caused by a novel mutation of the surfactant protein B gene and misalignment of lung vessels in

366 consanguineous kindred infants. Eur J Pediatr. 1999;158:513-8.

367 35. Haq K, McElhaney JE. Ageing and respiratory infections: The airway of ageing. Immunol Lett. Elsevier;

$368 \quad 2014 ; 162: 323-8$.

369 36. Ober C, Loisel DA, Gilad Y. Sex-specific genetic architecture of human disease [Internet]. Nat. Rev.

370 Genet. Nature Publishing Group; 2008 [cited 2020 Jul 8]. p. 911-22. Available from:

371 www.nature.com/reviews/genetics

372 37. Falagas ME, Mourtzoukou EG, Vardakas KZ. Sex differences in the incidence and severity of

373 respiratory tract infections. Respir. Med. W.B. Saunders; 2007. p. 1845-63.

374 38. Rivellese F, Prediletto E. ACE2 at the centre of COVID-19 from paucisymptomatic infections to severe 375 pneumonia. Autoimmun. Rev. Elsevier B.V.; 2020.

376 39. Piras V, Selvarajoo K. Beyond MyD88 and TRIF pathways in Toll-like receptor signaling. Front 377 Immunol [Internet]. Frontiers Research Foundation; 2014 [cited 2020 Jun 24];5. Available from:

378 www.frontiersin.org

379 40. Choudhury A, Mukherjee S. In silico studies on the comparative characterization of the interactions 380 of SARS-CoV-2 spike glycoprotein with ACE-2 receptor homologs and human TLRs. J Med Virol. John 381 Wiley and Sons Inc.; 2020;

382 41. Gong D, Farley K, White M, Hartshorn KL, Benarafa C, Remold-O'Donnell E. Critical role of SerpinB1 in 383 regulating inflammatory responses in pulmonary influenza infection. J Infect Dis [Internet]. 2011 [cited

3842020 Jun 24];204:592-600. Available from: https://academic.oup.com/jid/article-

385 abstract/204/4/592/2192600

386 42. Benarafa C, LeCuyer TE, Baumann M, Stolley JM, Cremona TP, Remold-O'Donnell E. SerpinB1 
protects the mature neutrophil reserve in the bone marrow. J Leukoc Biol. Wiley-Blackwell; 2011;90:21-

9.

43. Hersh CP, Campbell EJ, Scott LR, Raby BA. Alpha-1 antitrypsin deficiency as an incidental finding in clinical genetic testing. Am. J. Respir. Crit. Care Med. American Thoracic Society; 2019. p. 246-8.

44. Tasch JJ, McLaughlan AT, Nasir AA. A novel approach to screening for alpha-1 antitrypsin deficiency: Inpatient testing at a teaching institution. Chronic Obstr Pulm Dis. COPD Foundation; 2018;5:106-10. statement 11 Medical and Health Sciences 1102 Cardi. Multidiscip. Respir. Med. BioMed Central Ltd.;

3972018.

46. Ni L, Zhou L, Zhou M, Zhao J, Wang DW. Combination of western medicine and Chinese traditional patent medicine in treating a family case of COVID-19. Front Med [Internet]. 2020 [cited 2020 Jun 24];14:210-4. Available from: https://doi.org/10.1007/s11684-020-0757-x minimally invasive autopsies]. Zhonghua bing li xue za zhi = Chinese J Pathol [Internet]. 2020 [cited 2020 Jun 24];49:E009. Available from: http://www.ncbi.nlm.nih.gov/pubmed/32172546 
409 
411 Table 1. Summary of pathways and diseases associated with blood ADE genes

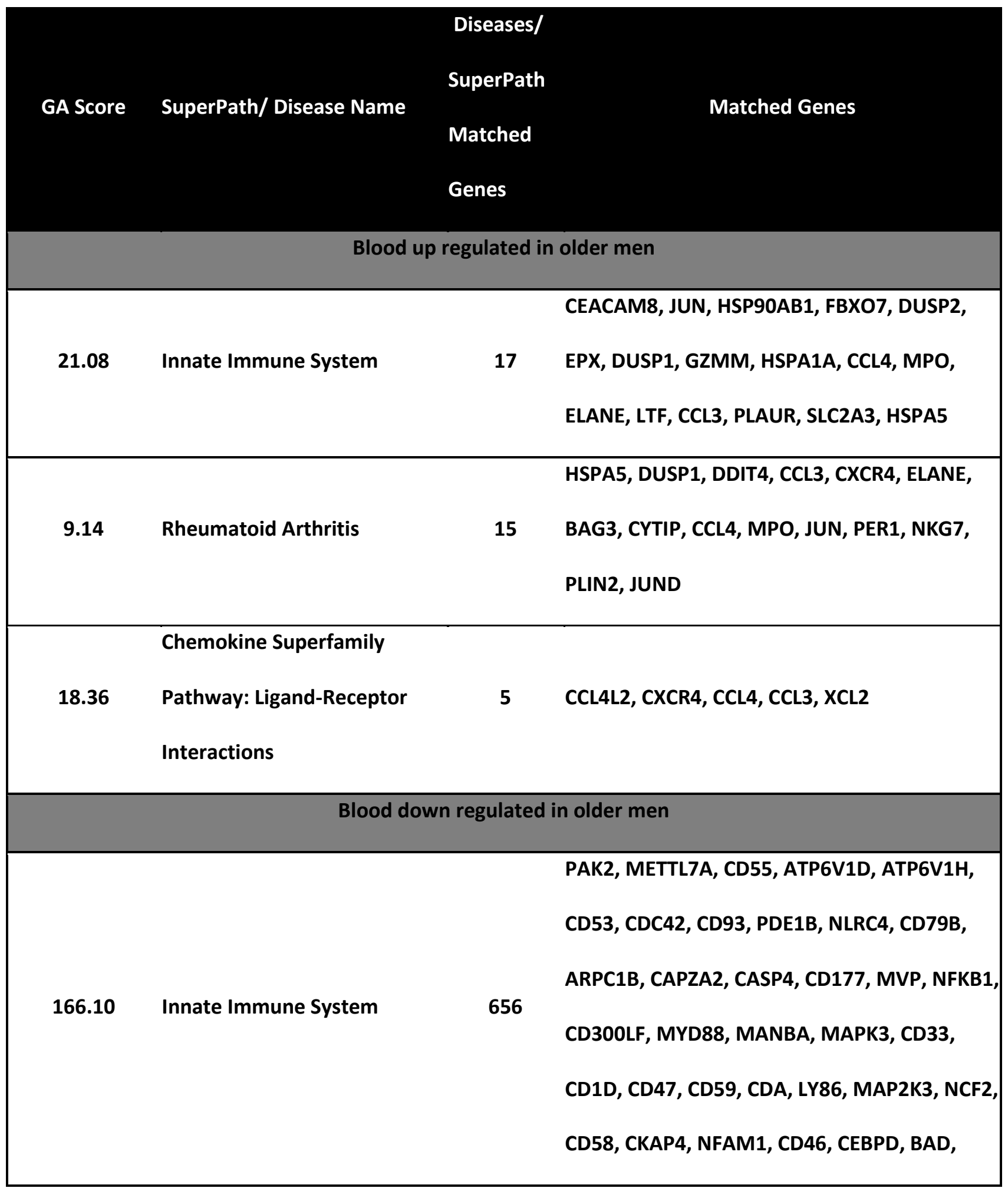




\begin{tabular}{|c|c|c|c|}
\hline & & & $\begin{array}{l}\text { BCL10, LTB, CCR1, PDXK, PIAS1, PECAM1, } \\
\text { MMP25, FBXW2, LYZ, MEFV, FGR [and sup. } \\
\text { Table 8] }\end{array}$ \\
\hline 49.83 & $\begin{array}{l}\text { Class I MHC Mediated Antigen } \\
\text { Processing and Presentation }\end{array}$ & 226 & $\begin{array}{l}\text { CTSB, CUL3, ICAM3, CLTC, CD36, FBXW5, IRS2, } \\
\text { LAIR1, IFI30, IFITM1, GRB2, LILRB4, GSK3A, } \\
\text { FBXL3, FBX040, FBX09, ITGB2, FBXO30, FGF8, } \\
\text { AG01, FCGR1A, CD274, FBXL5, ANAPC13, } \\
\text { AP2S1, CSK, KLHL2, ACTR1A, ANAPC5, KLHL21, } \\
\text { CTSA, CUL2, FCGR1B, LILRB3, LILRA6, CD14, } \\
\text { CD300LD, CTSK, AP1M2, KBTBD7, AGO4, CTSC, } \\
\text { ASB7, LILRA5 }\end{array}$ \\
\hline 35.08 & $\begin{array}{l}\text { Antigen Processing-Cross } \\
\text { Presentation }\end{array}$ & 54 & $\begin{array}{l}\text { CD36, FCGR1A, FCGR1B, CD14, CYBA, B2M, } \\
\text { BTK, CTSS, MYD88, NCF2, LY96, NCF4, NCF1, } \\
\text { PSMA6, PSMD13, PSMB7, PSMC2, TIRAP, } \\
\text { TLR4, PSMB1, PSMD6, TLR1, PSMA1, PSMC1, } \\
\text { TLR2 [and sup. Table 8] }\end{array}$ \\
\hline 31.03 & $\begin{array}{l}\text { Toll-like Receptor Signaling } \\
\text { Pathway }\end{array}$ & 112 & $\begin{array}{l}\text { CREB5, BIRC2, CFLAR, FBXW5, CISH, JAK3, } \\
\text { CUEDC2, IFNAR1, IL18, EIF2AK1, GRB2, IRAK4, } \\
\text { FADD, CLDN9, IRAK3, IRF9, GNAI3, CASP10, } \\
\text { IFNAR2, CTNNB1, CD14, CTSK, APAF1, IFNGR1, } \\
\text { GNAI2, GNAQ, DDX3X, ARAF, EIF2AK2, DDX58, } \\
\text { IFNK, BID, CLDN11, GSK3B, BTK, E2F3, CASP3, } \\
\text { MAPK13, MAPK14, IL1B[and sup. Table 8] }\end{array}$ \\
\hline
\end{tabular}




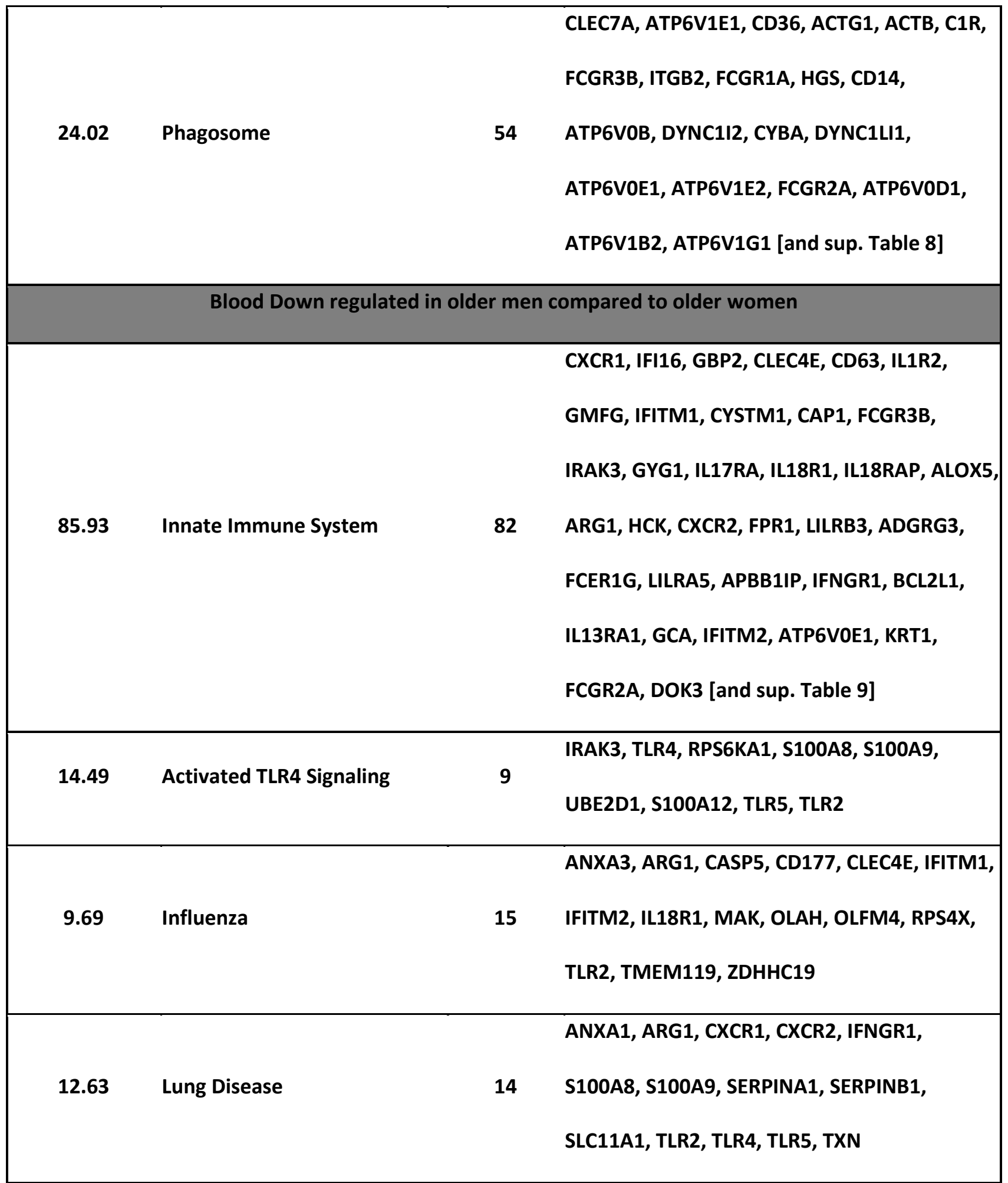




\begin{tabular}{|c|c|c|c|}
\hline \multicolumn{2}{|c|}{ GA top Scores SuperPath/ Disease Name } & seas & S/ SuperPath $\quad$ Matched Genes \\
\hline \multicolumn{4}{|c|}{ Lung up regulated in older men } \\
\hline 10.39 & Defensins & 3 & DEFA5, ITLN1, CAMP \\
\hline 5.31 & $\begin{array}{l}\text { Diabetes Mellitus, Noninsulin- } \\
\text { Dependent }\end{array}$ & 7 & $\begin{array}{l}\text { APOA2, ESM1, ITLN1, GH1, SCT, REG1B, } \\
\text { REG1A }\end{array}$ \\
\hline 4.78 & Crohn's Disease & 5 & HCAR3, REG1A, REG1B, IFNG, DEFA5 \\
\hline \multicolumn{4}{|c|}{ Lung down regulated in older men } \\
\hline 58.50 & Surfactant Metabolism & 11 & $\begin{array}{l}\text { ABCA3, DMBT1, CTSH, PGA4, NAPSA, } \\
\text { SFTPB, SFTPD, SFTPC, SLC34A2, SFTPA2, } \\
\text { SFTPA1 }\end{array}$ \\
\hline 31.27 & $\begin{array}{l}\text { Defective CSF2RA Causes Pulmonary } \\
\text { Surfactant Metabolism Dysfunction } \\
4 \text { (SMDP4) }\end{array}$ & 5 & SFTPB, SFTPD, SFTPC, SFTPA2, SFTPA1 \\
\hline 13.50 & Pulmonary Fibrosis & 15 & $\begin{array}{l}\text { ABCA3, ALDH3A1, FMO5, CRTAC1, BPIFB1, } \\
\text { LAMP3, PEBP4, MFSD2A, PLA2G4F, PLA2G3, } \\
\text { SFTPA1, SFTPA2, SFTPC, SFTPD, ZNF385B }\end{array}$ \\
\hline 9.02 & Lung Disease & 10 & $\begin{array}{l}\text { SFTPA1, SFTPA2, SFTPB, SFTPC, SFTPD, SLPI, } \\
\text { NPPB, CFTR, ABCA3, MUC1 }\end{array}$ \\
\hline 8.78 & Respiratory Failure & 9 & $\begin{array}{l}\text { CFTR, ABCA3, NPPB, REN, FGA, NPPA, } \\
\text { SFTPC, SFTPD, SFTPB }\end{array}$ \\
\hline
\end{tabular}




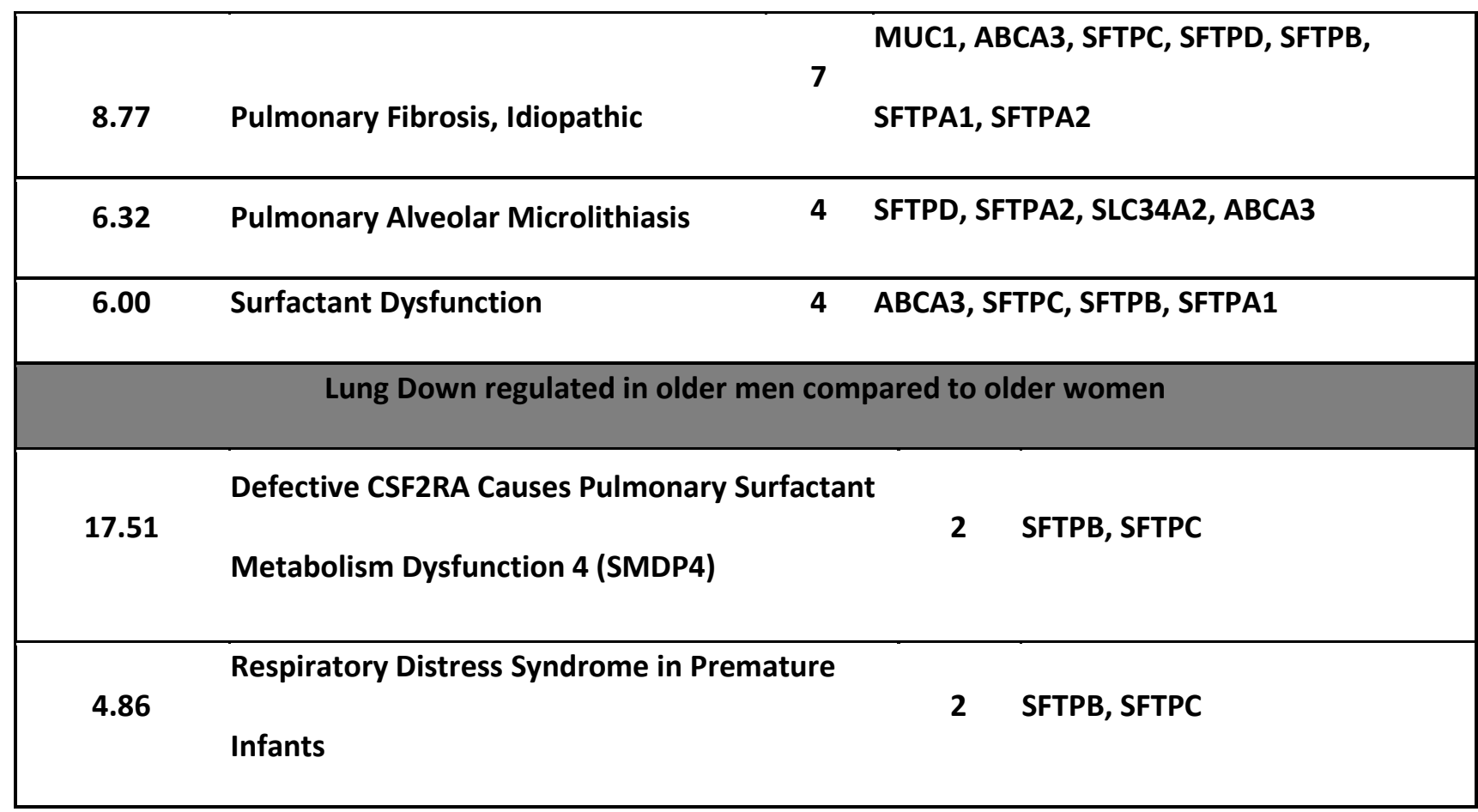

415 Table 3. Summary of genetic variants associated with Lung SFTPs genes expression and function.

\begin{tabular}{|c|c|c|}
\hline Gene & Top eQTLs hits & Top GWAS hits (phenotype) \\
\hline SFTPB & rs4832183; rs4832182 & - \\
\hline SFTPC & - & rs8192327 (FEV1/FVC) \\
\hline SFTPA1 & rs1650158 & rs2559728 (FEV1/FVC) \\
\hline SFTPA2 & rs1650158; rs35565917 & rs2559728 (FEV1/FVC) \\
\hline SFTPD & rs149407882 & $\begin{array}{l}\text { rs1923539-A; rs1923539-A; rs3923564-G } \\
\text { (Chronic obstructive pulmonary disease) }\end{array}$ \\
\hline
\end{tabular}

417 Figures legends 
418 Figure 1. Numbers of identified ADE genes in all analyses. Schematic representation of the DE genes

419 found between the tested conditions in the lung (a) and in the blood (b).

420 Figure 2. Expression of blood ADE genes. Distribution of gene expression levels of blood ADE genes, in

421 women older than 60 (pink), women younger than 60 (red), men older than 60 (light blue), and men

422 younger than 60 (blue). The TPM values are presented on the $\mathrm{Y}$ - axis.

423 Figure 3. Expression of lung ADE genes. Distribution of gene expression levels of lung ADE genes, in

424 women older than 60 (pink), women younger than 60 (red), men older than 60 (light blue), and men

425 younger than 60 (blue). The TPM values are presented on the $Y$ - axis.

426 Figure 4. Surfactants genes expression tend to be correlate with the ADE genes of the innate immune

427 system. The Correlation matrix as a heatmap, between the lung SFTPs genes (X-axis) and the DE innate

428 immune genes between elderly men to elderly women ( $Y$ - axis). The matrix is sorted according to the

429 highest correlation scores between the innate immune ADE genes to lung SFTPB. 
Figures

a.

Men age related

Women age related

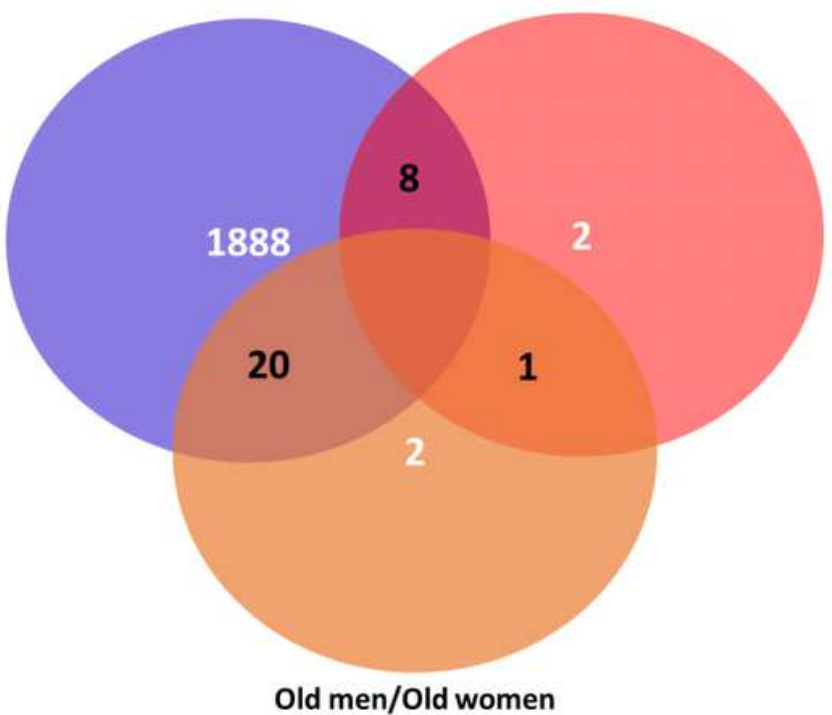

b.

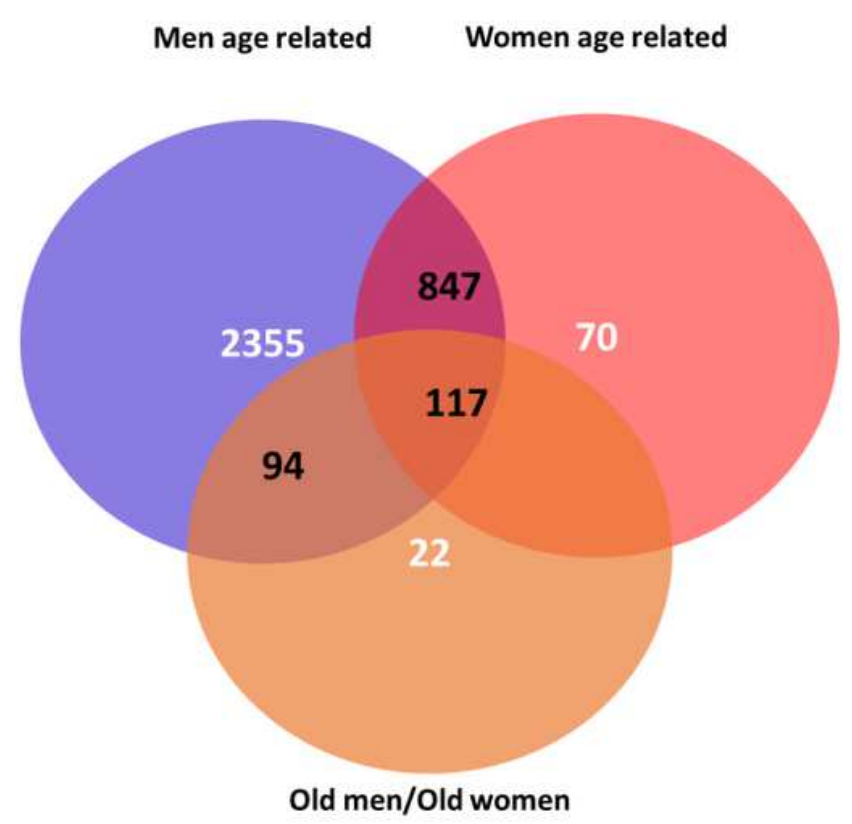

\section{Figure 1}

Numbers of identified ADE genes in all analyses. Schematic representation of the DE genes found between the tested conditions in the lung (a) and in the blood (b).
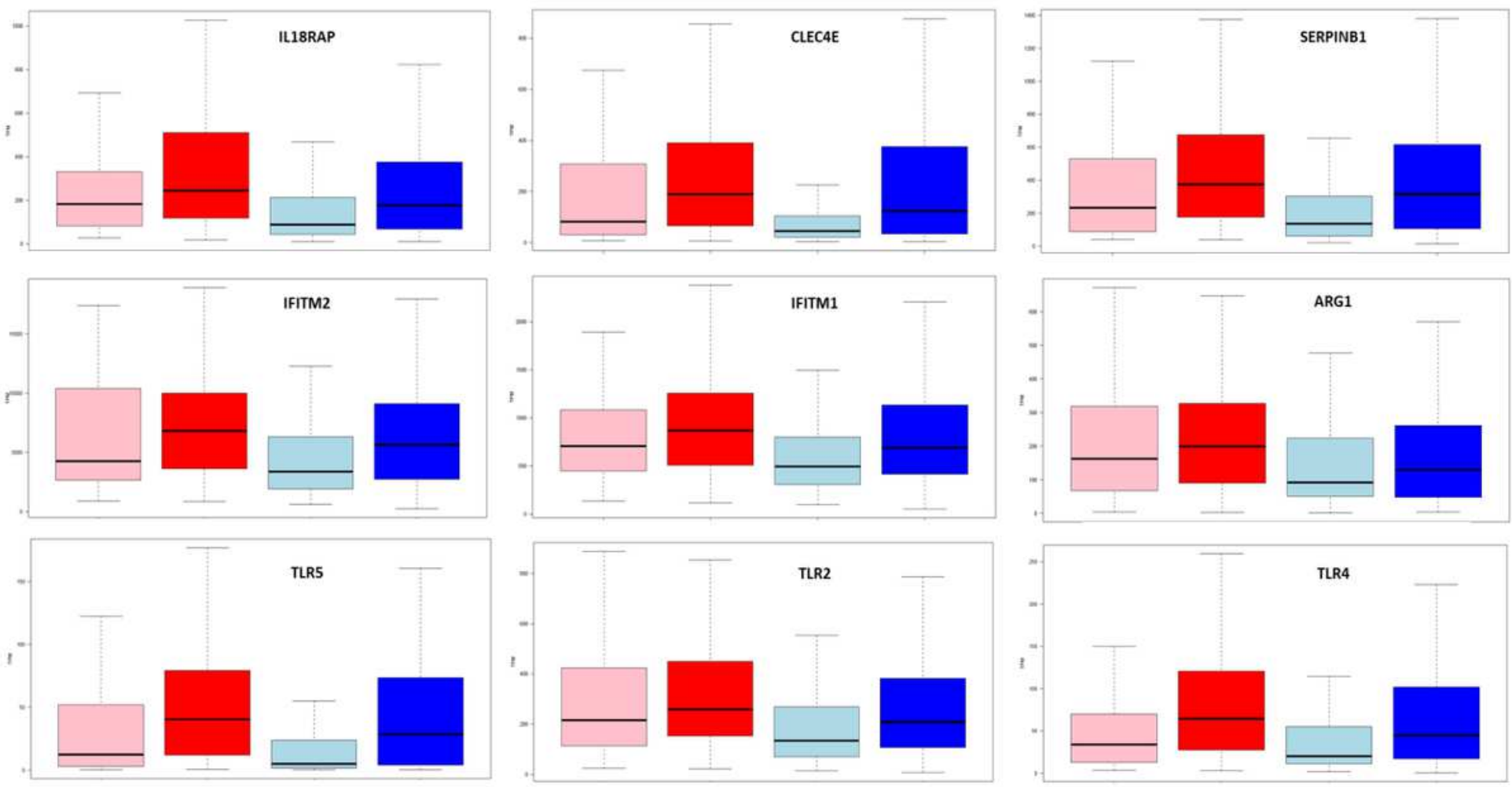

Figure 2 
Expression of blood ADE genes. Distribution of gene expression levels of blood ADE genes, in women older than 60 (pink), women younger than 60 (red), men older than 60 (light blue), and men younger than 60 (blue). The TPM values are presented on the Y-axis.
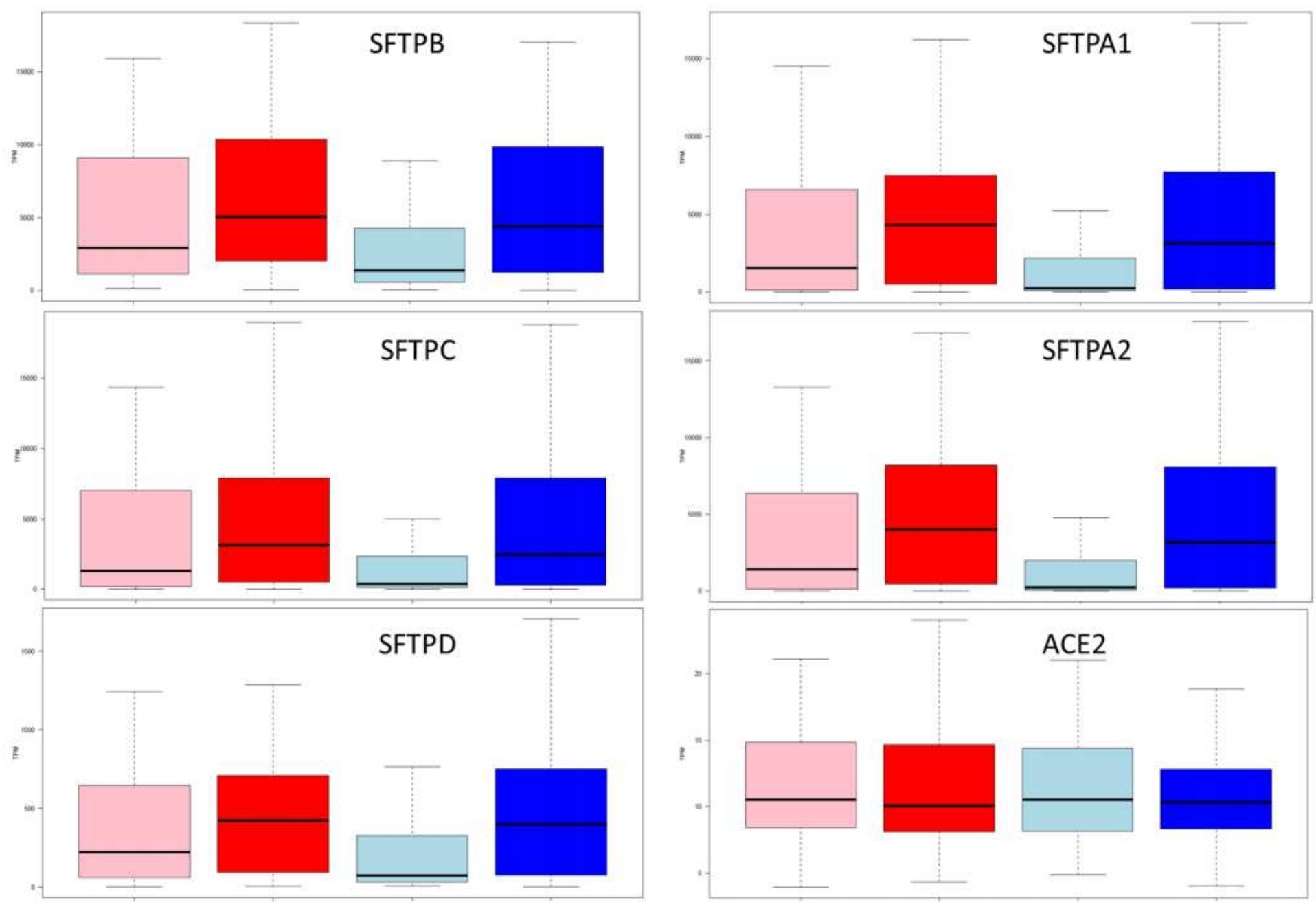

\section{Figure 3}

Expression of lung $A D E$ genes. Distribution of gene expression levels of lung $A D E$ genes, in women older than 60 (pink), women younger than 60 (red), men older than 60 (light blue), and men younger than 60 (blue). The TPM values are presented on the $Y$ - axis. 


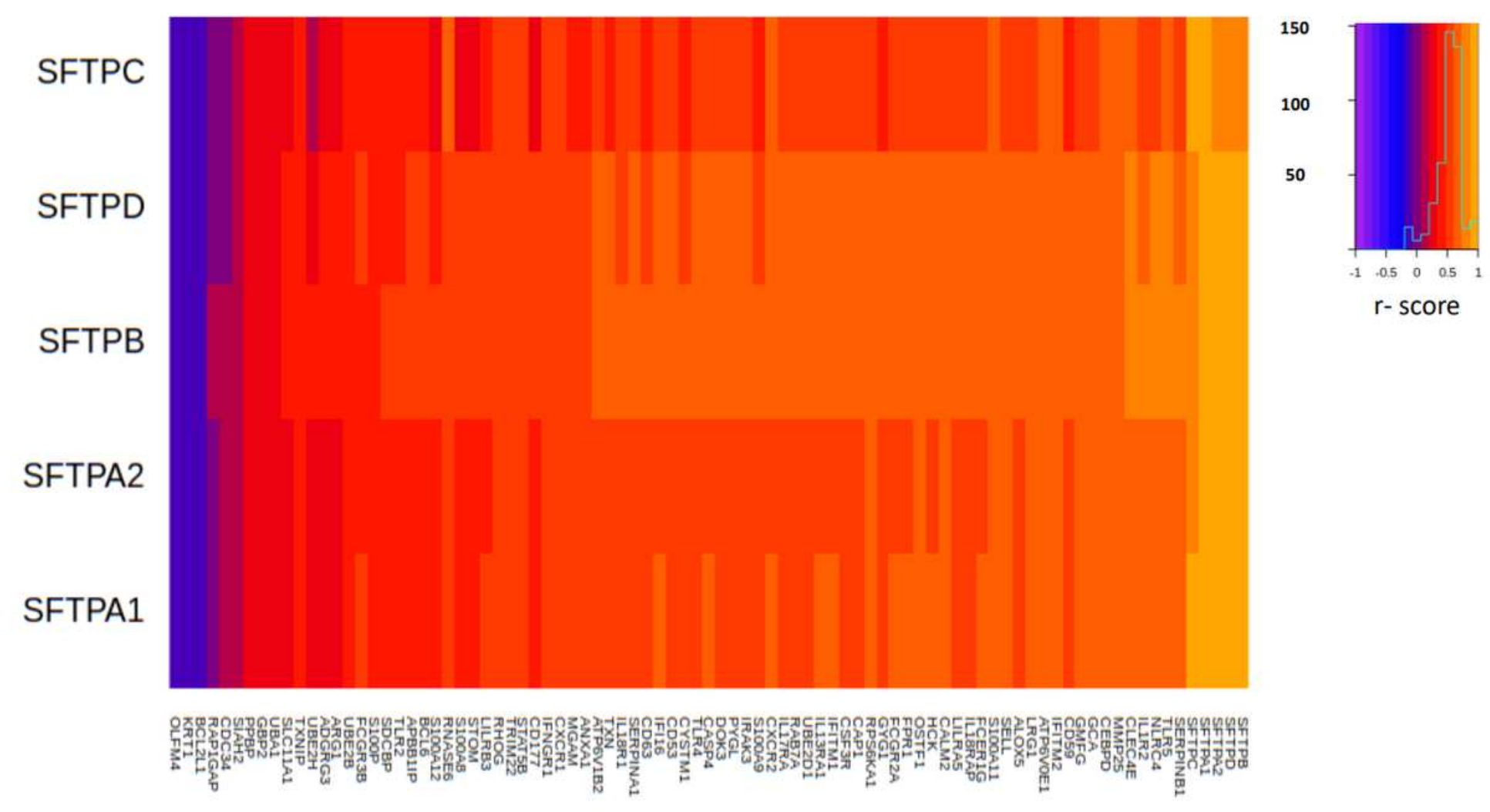

Figure 4

Surfactants genes expression tend to be correlate with the ADE genes of the innate immune system. The Correlation matrix as a heatmap, between the lung SFTPs genes (X-axis) and the DE innate immune genes between elderly men to elderly women ( $\mathrm{Y}$ - axis). The matrix is sorted according to the highest correlation scores between the innate immune ADE genes to lung SFTPB.

\section{Supplementary Files}

This is a list of supplementary files associated with this preprint. Click to download.

- Supp.table7.GeneAnalyticsBloodmenUpregulated.xlsx

- Supp.table1.Blood.Male.ADE.csv

- Supp.table8.GeneAnalyticsBloodmenDownregulated.xlsx

- Supp.table3.Blood.oldmen.oldwomen.DE.csv

- Supp.table9.GeneAnalyticsBlood.oldmen.oldwomen.xlsx

- Supp.table10.GeneAnalyticsLung.menupregulated.xIsx

- Supp.table2.Blood.Female.ADE.csv

- Supp.table11.GeneAnalyticsLung.mendownregulated.xlsx

- Supp.table4.Lung.Male.ADE.csv

- Supp.table12.GeneAnalyticsLung.oldmen.oldwomen.xlsx 
- Supp.table6.Lung.oldmen.oldwomen.DE.csv

- Supp.table5.Lung.Female.ADE.csv 